\title{
Encoding by control of the symbolic dynamics emitted by a chaotic laser
}

\author{
Juan Carlos Martín* \\ Department of Applied Physics and I3A, University of Zaragoza, Pedro Cerbuna 12, 50009 Zaragoza, Spain
}

(Received 21 May 2014; revised manuscript received 14 October 2014; published 17 February 2015)

\begin{abstract}
Application to a chaotic erbium-doped fiber laser of the digital encoding technique by control of its emitted symbolic dynamics is numerically tested. Criteria to select the better working conditions and the perturbation to be introduced in any control parameter are proposed. Once they are chosen, the procedure to prepare the system for control and the way to carry it out are described. It is shown that the general method cannot be blindly applied, but it must be adapted to the particular case under analysis for a good performance. Finally, in relation to a possible experimental implementation, influence of noise in the bit error rate of the communication system is discussed.
\end{abstract}

DOI: 10.1103/PhysRevE.91.022914

PACS number(s): 05.45.Gg, 05.45.Vx, 42.55.Wd

\section{INTRODUCTION}

Since the early 1990s, the application of chaos for communication purposes is a very active subject of research. Especially, chaos synchronization techniques have been widely studied for application to secure communications since the initial suggestion of this idea by Pecora and Carroll [1] and the subsequent experiment by Cuomo and Oppenheim [2]. Besides, it has been shown that lasers are excellent devices to apply these techniques to them [3-5], which has led to the proposal of several optical secure communication systems. On the other hand, Hayes and collaborators proposed another possible application of chaos for communication purposes: They demonstrated how a chaotic circuit could be forced to emit a signal containing a predefined sequence of binary symbols [6,7] by means of chaos control techniques based on small perturbations [8]. This way, feasibility of a digital communication procedure based on a chaotic system was shown. Besides, this procedure presents a remarkable virtue: As the control is exerted by means of small perturbation signals, it requires very low energy consumption. Since then, reports on applications of this technique to other chaotic systems are very scarce. As far as I know, it has only been applied to a chemical system in which the BelousovZhabotinsky reaction takes place [9] and to a chaotic traveling wave tube microwave generator [10].

The aim of this paper is to analyze whether this technique provides good results when applied to a chaotic erbium-doped fiber laser (EDFL). The study is not only intended to broaden the scope of possible systems in which the Hayes method can work successfully, but more specifically to analyze its implementation in a device especially suited for communication purposes such as an EDFL, whose integration within an optical communications network is, obviously, totally natural. The study is numerical and it relies on recent results that prove that the EDFL model employed here provides accurate predictions of the EDFL behavior, even in a chaotic regime [11].

The Hayes procedure to generate a desired sequence of symbols counts on a solid basis that guarantees good results when applied to mathematical systems characterized by continuous,

*jcmartin@unizar.es univalued, one-dimensional first-return maps. Nevertheless, physical systems present some features that may become serious impairments when applying this control procedure: Their first-return maps are not strictly univalued and they are affected by noise (a specific treatment of noise influence on encoding procedures based on chaotic systems is thoroughly developed in Ref. [12]). Besides, with a mathematical system there is no problem in introducing a perturbation in any control parameter, no matter the range of values of the perturbation nor the accuracy required. But, obviously, in a physical system some parameters cannot be easily modified, so that considering them as control parameters is not realistic. Moreover, in the case that they lend themselves to be modified, it is not at all evident that they can be adjusted with the accuracy required. On the other hand, after perturbation the system response needs a relaxation time to get back to its attractor. This method works if between two consecutive perturbations the system has enough time to relax almost completely. The aim of this paper is testing whether the method can work realistically in an erbium-doped fiber laser: whether the control can be carried out by using a realistic control parameter with realistic perturbation values and in the presence of noise. (And, still, an important aspect would remain to be analyzed for a realistic implementation of the method: whether the perturbation can be calculated and generated fast enough to be applied once per system modulation period; but this is purely a question on electronics and informatics and it exceeds the scope of this work.)

The structure of the paper is as follows. Section II is a brief summary of the Hayes method, in which I have also included the presentation of some basic concepts, aimed at readers unfamiliar with the subject. In Sec. III, the chaotic system employed is described: First, its equations are presented and the particular EDFL considered is stated, taking into account parameters belonging to a real EDFL; and second, the selection process of the particular working conditions is explained. Section IV is devoted to describing the implementation of the Hayes method to the EDFL. As it will be shown, a blind implementation does not give good results: A customization process to the particular system under study is necessary to get a good performance. The approach intends to be as realistic as possible and that is why the influence of noise in the controllability of the system is analyzed 
in Sec. V. Finally, the paper ends with the Conclusions section.

\section{BASIC CONCEPTS}

This section is devoted to providing the outlines of the concepts involved in the technique of encoding by feedback control of the symbolic dynamics emitted by a chaotic system. For a much more precise and detailed analysis, I recommend Bollt's review [13].

The behavior of any nonlinear system is usually described by a relation such as

$$
\vec{X}_{N+1}=f\left(\vec{X}_{N}\right)
$$

where $f$ is a map of a state space into itself and $\vec{X}_{N}$ denotes the state coordinates at the discrete instant $t_{N}$. Continuous-time dynamical systems also admit this description: A standard resource for their analysis is the use of first-return maps, that is to say, maps relating consecutive intersections of the system's trajectory with a certain Poincaré section (PS). In low-dimensional, highly dissipative systems, intersections with PSs can be often parametrized so that approximated univalued, one-dimensional first-return maps are obtained $[14,15]$.

Figure 1 represents a first-return map in which a partition with four regions has been established. An orbit or sequence of $X$ values generated by the map departing from $X_{0}$ will be denoted here by $O\left(X_{0}\right)=\left\{X_{i}\right\}_{i=0}^{\infty}=X_{0} X_{1} X_{2} X_{3} X_{4} \cdots$. Associated to any of these sequences, there is also a symbolic sequence $S\left(X_{0}\right)=\left\{K_{i}\right\}_{i=0}^{\infty}$, where $K_{i}$ is the symbol assigned to the interval to which $X_{i}$ belongs (in the example in Fig. 1, this sequence is $31233 \cdots)$. As it is well known $[14,16]$, the partition is said to be generating if there is a one-to-one correspondence between abscissa points and symbolic sequences. In particular, if a map is a continuous, nonmonotonous application $f$ of an interval $\left[X_{\mathrm{MIN}}, X_{\mathrm{MAX}}\right.$ ] into itself (as in Fig. 1), a generating partition can be obtained by dividing the interval into the different subintervals separated by the critical points $X_{\mathrm{CR}, i}$, that is to say, points such that $f^{\prime}\left(X_{\mathrm{CR}, i}\right)=0$, where $i=1,2, \ldots, n, n$ is the number of critical points in the map [16,17]. Here, each of these subintervals will be referred to as a sector. Under the above-

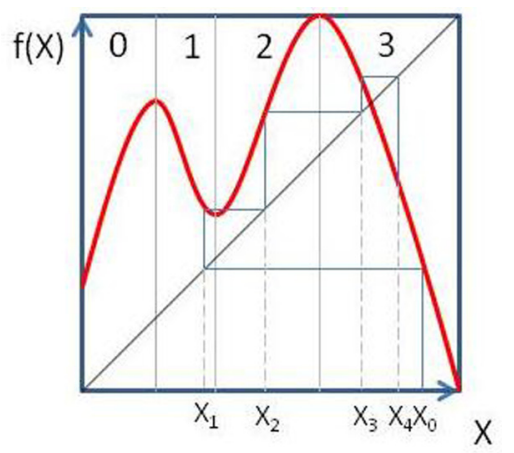

FIG. 1. (Color online) Example of a first-return map. Each monotonous region constitutes a region of the generating partition. The first terms of the sequence departing from $X_{0}$ are graphically calculated. mentioned conditions, kneading theory holds [18] and an ordering $(\prec)$ between symbolic sequences can be defined: $S\left(X_{0}\right) \prec S\left(Y_{0}\right)$ if $X_{0}<Y_{0}[19]$.

Assume $X_{i}<Y_{i}$, both belonging to the same sector of the generating partition. If the first-return map is increasing within this sector, then $X_{i+1}<Y_{i+1}$ and the sector is said to be "orientation preserving" or "even." On the other hand, if the sector is decreasing, then $Y_{i+1}<X_{i+1}$, and the sector is said to be "orientation reversing" or "odd." This simple concept is the foundation of the rule that allows one to order symbolic sequences without knowledge of their starting abscissa values. Suppose that, as usual, sectors are labeled from left to right by consecutive, ascending, non-negative integer numbers, chosen so that the parity of the labels matches the "parity" of the sectors. Given two different symbolic sequences, $S_{A}=\left\{K_{i}\right\}_{i=0}^{\infty}$ and $S_{B}=\left\{J_{i}\right\}_{i=0}^{\infty}$, with $K_{i}=J_{i}$ for $i<p$ and $K_{p} \neq J_{p}, S_{A} \prec S_{B}$ if $(-1)^{m} \operatorname{sgn}\left(J_{P}-K_{P}\right)=1$ and $S_{B} \prec S_{A}$ otherwise, $m$ being the number of odd symbols in the sequence $\left\{K_{i}\right\}_{i=0}^{p-1}$.

While an infinite symbolic sequence defines an abscissa point, a finite symbolic sequence defines an interval of the abscissa axis: we can naturally talk about "the interval $\left\{K_{i}\right\}_{i=0}^{n}$ " to mean "the interval of $X$ values such that the first $n$ values of their symbolic sequences are $\left\{K_{i}\right\}_{i=0}^{n}$." Figure 2 illustrates this idea: All intervals defined by two symbols within the 0 interval are pointed out in the first-return map employed as an example. Besides, Fig. 2 is useful to illustrate a concept which is very relevant in this work and central in information theory [20]: the concept of forbidden sequence. It can be appreciated that the map does not present any 00 interval. Therefore, the map does not generate any sequence containing 00 . In information theory, the sequences that the system does not generate are called forbidden sequences and the rules that establish allowed and forbidden sequences are called the grammar, which can be determined by application of a systematic method (see [21,22] for a general description, [23] for an analysis centered on chaotic systems, or $[9,12]$ for a more specific study aimed at encoding by chaos control).

The key idea of the procedure proposed in Refs. [6,7] for encoding is that by perturbing any control parameter, the first-return map becomes momentarily moved so that the image of any abscissa point becomes modified (Fig. 3) and, therefore, so does the subsequent sequence. The movement is not homogeneous, as Fig. 3 illustrates: The image of each

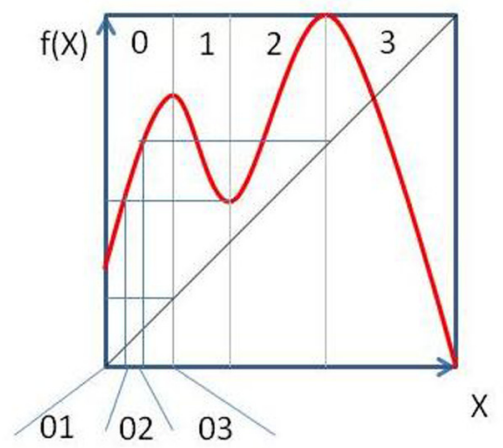

FIG. 2. (Color online) Graphical determination of intervals defined by two symbols within the 0 region. 


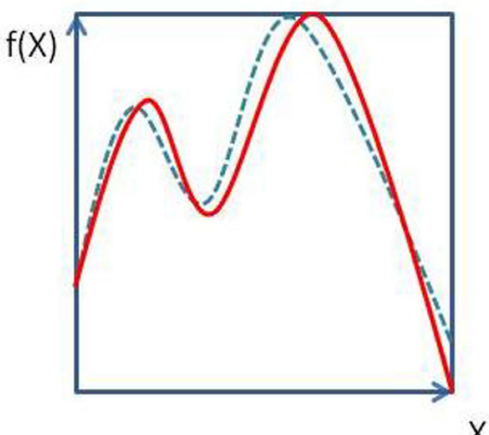

FIG. 3. (Color online) Sketch of the way the original first-return map (solid line) characteristic of the system may become momentarily modified by means of a perturbation, giving rise to a slightly different behavior described by a provisional first-return map (dashed line).

abscissa point changes by a different amount. However, if the perturbation range is small enough, displacement of the image of each abscissa point as a function of the perturbation can be approximated by a characteristic straight line that can be calibrated. So, by selecting a suitable amount of the perturbation, the image of a given abscissa can be chosen, at least within a small range in the neighborhood of its unperturbed image. So, instead of $O\left(X_{0}\right)=X_{0} \mathrm{X}_{1} \mathrm{X}_{2} \ldots$, where $X_{i}=f\left(X_{i-1}\right)$, the perturbation gives rise to $O^{\prime}\left(X_{0}\right)=X_{0}^{\prime} X_{1}^{\prime} X_{2}^{\prime} \ldots$, where $X_{1}^{\prime}=X_{1}+\varepsilon$ and $X_{2}^{\prime}$ can be considered equal to $f\left(X_{1}^{\prime}\right)$ if the perturbation is small enough to allow fast enough system relaxation to its attractor.

This means that by application of a perturbation, a symbolic sequence within the neighborhood of the one corresponding to the unperturbed system can be chosen. Of course, the choice has limited accuracy. Suppose that the system's accuracy allows one to know the first $N$ symbols of $S\left(X_{0}\right)$, once $X_{0}$ has been given: $S\left(X_{0}\right)=K_{0} K_{1} K_{2} \ldots K_{N-1} K_{N} \ldots$, where all $K_{i}(i=1, N)$ are known. Suppose also that the system admits a calibration such that it is possible to calculate the perturbation necessary to shift slightly $f\left(X_{0}\right)$ so that symbols $K_{0}$ to $K_{N-1}$ remain and $K_{N}$ can be chosen. Under these assumptions, it is possible to force the system to emit any sequence of symbols (at least any allowed sequence) from the $N$ th symbol on: $f\left(X_{0}\right)$ is perturbed to obtain the desired $K_{N}, f\left(X_{1}+\varepsilon\right)$ which is again perturbed to obtain the desired $K_{N+1}$, and so on.

\section{THE CHAOTIC SYSTEM EMPLOYED}

\section{A. Model}

The scheme of a typical unidirectional EDFL ring setup can be found in Ref. [24], in which a laser model is shown to predict successfully the resonance frequencies of the system, multistable behavior working conditions, etc., for sine-wave modulation of the laser's pump power. The model consists of a nonautonomous system of two equations:

$$
\begin{gathered}
\frac{d N_{2 r}(t)}{d t}=S_{1}(t)-S_{2}(t) N_{2 r}(t)+S_{3} P_{l}(t)-S_{4} P_{l}(t) N_{2 r}(t), \\
\frac{d P_{l}(t)}{d t}=P_{l}(t)\left[R_{1}+R_{2} N_{2 r}(t)\right],
\end{gathered}
$$

where

$$
\begin{gathered}
S_{1}(t)=\frac{\gamma_{a}\left(v_{p}\right)}{h v_{p} N_{t}} P_{p}(t), \\
S_{2}(t)=\frac{\gamma_{a}\left(v_{p}\right)+\gamma_{e}\left(v_{p}\right)}{h v_{p} N_{t}} P_{p}(t)+\frac{1}{\tau}, \\
S_{3}=\frac{\gamma_{a}\left(v_{l}\right)}{h v_{l} N_{t}}, \\
S_{4}=\frac{\gamma_{a}\left(v_{l}\right)+\gamma_{e}\left(v_{l}\right)}{h v_{l} N_{t}}, \\
R_{1}=\frac{c}{D}\left[\operatorname{Ln}(T)-\gamma_{a}\left(v_{l}\right) L\right], \\
R_{2}=\frac{c L}{D}\left[\gamma_{a}\left(v_{l}\right)+\gamma_{e}\left(v_{l}\right)\right] .
\end{gathered}
$$

In the preceding coefficients, $t$ is the time parameter, $h$ is the Planck constant, $N_{T}$ is the number of $\mathrm{Er}^{3+}$ ions per unit length, $\tau$ is the laser transition lifetime, $v_{p}$ and $v_{l}$ are the pump and laser signal optical frequencies, $\gamma_{a}$ and $\gamma_{e}$ represent the absorption and emission coefficients, $T$ is the one-trip transmission coefficient of the resonator ring, $L$ is the active medium length, $c$ is the speed of light in vacuum, and $D$ is the ring optical path. $P_{p}$ is the pump power, in this case with a cosine wave profile: $P_{p}(t)=P_{p 0}(1+m \cos \phi t)$, where $P_{p 0}$ stands for average pump power, $m$ is the modulation index, and $\phi$ is the modulation phase: $\phi(t)=2 \pi[f t-I N T(f t)], f$ being the modulation frequency.

In this system, the explicit state variables are $N_{2 r}$ and $P_{l}$, which represent, respectively, the population of the upper laser transition level and the power of the laser signal averaged over the active medium. In the first equation, the first two contributions to $d N_{2 r}(t) / d t$ account for pump absorption, pump stimulated emission, and spontaneous emission, while the last two terms account for laser signal absorption and laser signal stimulated emission. The second equation accounts for the phenomena that cause laser signal power variations: the losses due to the cavity passive elements (contained in $R_{1}$ ) and the amplification provided by the active medium.

The EDFL ring chaotic behavior induced by sine-wave modulation of the laser's pump power has been thoroughly analyzed in Ref. [25]. The experimental results presented there show that depending on the modulation frequency and on the modulation index, laser emission is ruled by chaotic attractors with different topological structures: horseshoe, reverse horseshoe, spiral, staple, or S. Note that branches in the template of a chaotic attractor amount to regions of the generating partition $[14,15]$. Capability of the model just presented for prediction of these behaviors was also successfully tested [11]. This reference guarantees reliability of the numerical results to be presented here, especially taking into account that the laser considered here has the same characteristic parameters as in Ref. [11].

\section{B. Selection of the working conditions}

The encoding technique to be employed here becomes facilitated if a generating partition is available $[13,26]$. There 


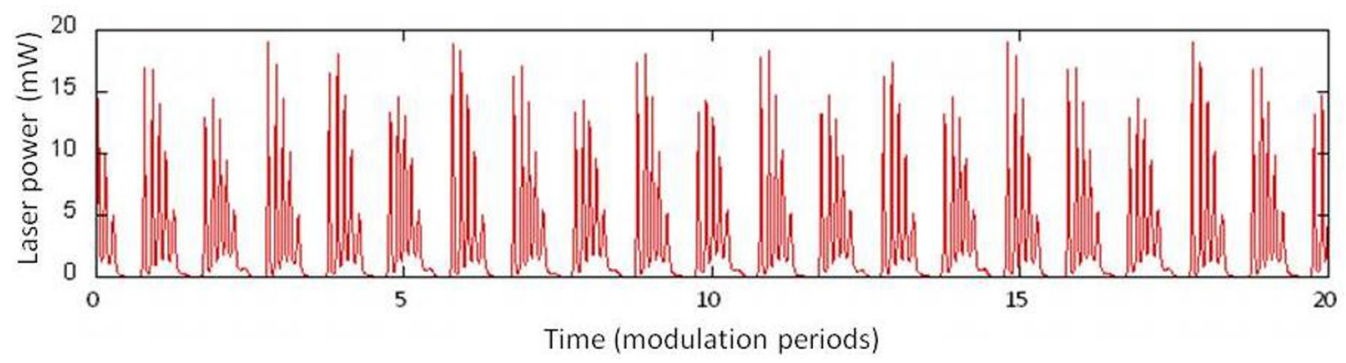

FIG. 4. (Color online) A temporal series emitted by the laser without perturbation.

is a good understanding about how to establish it in the cases of hyperbolic systems and noninvertible maps of an interval into itself [14,27-29]. Outside these two limiting cases, determination of a generating partition may become a challenging task (although there are techniques available [30,31]). In experimental systems such as the one studied here, an option to establish a partition (in general, not a generating one) is by means of threshold-crossing analysis [32]. Nevertheless, a partition established this way may lead to significant reduction in topological entropies and a high degree of nonuniqueness [13,26], both of them serious troubles for encoding purposes. Fortunately, the EDFL employed here is a low-dimensional, highly dissipative system and, therefore, it is possible to find PSs which can be parametrized so that approximated continuous, univalued, one-dimensional firstreturn maps are obtained $[14,15]$. In these cases, a partition can be established by dividing the domain of the map into the intervals between its different critical points, as previously remarked in Sec. II. Certainly a partition like this is not strictly generating, but in view of the similarity between the experimental maps and the ideal, one-dimensional maps, it can be expected that the behavior of the partition chosen will not be dramatically different from the behavior of a perfectly defined generating partition and, therefore, it will provide good results for encoding purposes (hypothesis to be confirmed or ruled out depending on the results obtained).

For selection of the working conditions to test the encoding method, different time series were generated with different combinations of the modulation parameters. According to the explanation given in the previous paragraph, a first criterion to choose suitable working conditions was to select the cases in which the PSs showed a cloud of points as similar to a line as possible. In these cases, the corresponding firstreturn maps were obtained following the method explained in Ref. [15]. This way, the number of regions in the corresponding generating partition became evident. For the sake of simplicity chaotic attractors with two regions (Smale horseshoes or reverse horseshoes) in their generating partition were preferred to attractors with more regions. Finally, the key feature to select the most suitable attractor was its spectrum of allowed sequences: the broader, the better for communication purposes. The case selected corresponds to an average pump power $P_{p 0}=43 \mathrm{~mW}$, modulation index $m=0.75$, and modulation frequency $f=3300 \mathrm{~Hz}$. From the point of view of a commercial application, frequency would be a key feature of the system: As it provides one bit per modulation period, modulation frequency coincides with the bits per second provided by the system. In this respect, the electronic system proposed in Ref. [6] is better, as it generates symbols at a faster speed $(f=5000 \mathrm{~Hz}$ ). Anyway, we are dealing with minuscule speed transmissions compared with the ones characteristic of commercial digital links.

Figure 4 shows a temporal series emitted by the laser without any added perturbation. Figure 5 shows several Poincaré sections constructed by time-delay embedding [14], $P_{l}(t+\Delta)$ against $P_{l}(t)$, for instants $t$ corresponding to different modulation phases. As this paper is oriented as a study of feasibility of the encoding method in a real laser, it has been preferred to deal with a PS as experimental, even though the dynamical variable $N_{2 r}(t)$ has also been calculated. Nevertheless, in the lab, usually just $P_{l}(t)$ is registered because $N_{2 r}(t)$ variations are very difficult to measure: Fluorescence power emitted transversally to the active fiber is proportional to $N_{2 r}(t)$ and it can be easily measured, but as $N_{2 r}(t)$ variations
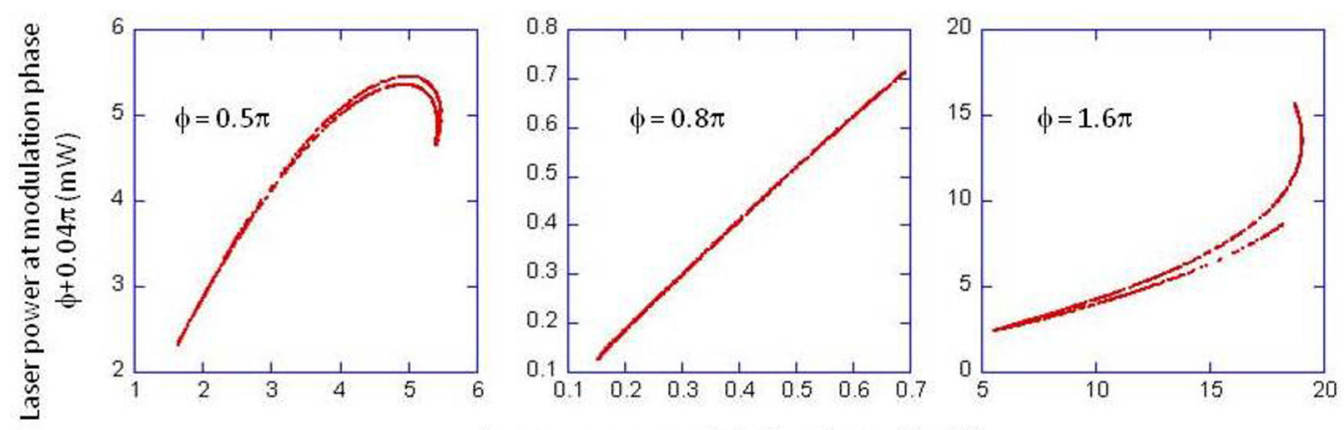

Laser power at modulation phase $\phi(\mathrm{mW})$

FIG. 5. (Color online) Poincaré sections for three laser pump modulation phases. 


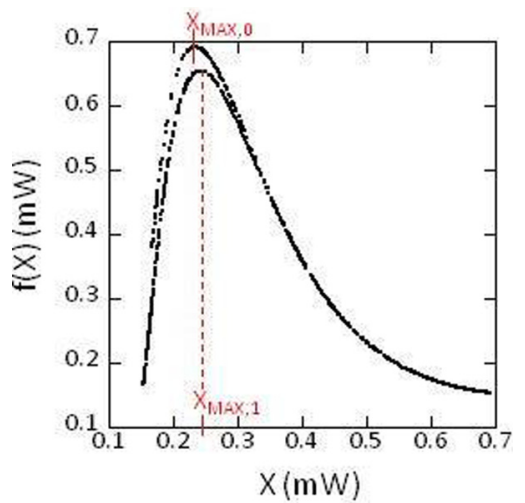

FIG. 6. (Color online) First-return map characteristic of the operating condition chosen.

are very small, fluorescence power variations are very difficult to appreciate and typically they become masked by the noise of the detector.

The PSs were calculated for 20 different modulation phases (although for illustration purposes, it has been considered sufficient to show just three of them in Fig. 5). Out of all these sections, the most convenient to get a first-return map is the one corresponding to the modulation phase $\phi=0.8 \pi$, called from now on "the measurement phase": As this PS can be approximated by a monotonous curve (almost a straight line), the first-return map can be obtained from it in a very straightforward way, by using its abscissa as a parameter (Fig. 6). From now on, we call this parameter $X$ and we will use the notation stated above both for sequences of $X$ values and for symbolic sequences generated by the map.

\section{CONTROLLED EMISSION OF SEQUENCES OF BITS}

\section{A. Preparing the system for control}

It can be observed that the resulting first-return map shown in Fig. 6 presents two layers, which upon close inspection reveal a double sublayer inside each of them, each sublayer having two sub-sublayers as well and so on, as is typical of this kind of system [23]. As will be justified by the results presented later, it is a good approximation to consider two layers in the map, without paying attention to their inner structure. This approximation implies to consider that the dynamical system behavior can be described by two functions, corresponding to the upper and lower layers, called here $f_{0}$ and $f_{1}$, respectively.

In order to establish correctly the limits of the 0 and 1 regions, a different treatment of both layers is crucial. As it can be seen in Fig. 6, maxima of both layers happen for two slightly different $X$ values. We call $X_{\mathrm{MAX}, 0}$ and $X_{\mathrm{MAX}, 1}$ the $X$ value so that $f_{0}(X)$ and $f_{1}(X)$ are maxima, respectively. It is considered that the system emits a 0 if $X$ has been emitted through layer 0 and it is less than $X_{\mathrm{MAX}, 0}$, or if $X$ has been emitted through layer 1 and it is less than $X_{\text {MAX,1 }}$. In any other case, the system emits a 1 (see Fig. 6). Whether the system's answer is given by the upper or the lower layer is not at all at random: By inspection of any $O(X)$ sequence, it is plain that $X_{N+1}=f_{0}\left(X_{N}\right)$ if $X_{N-1}$ belongs to the interval 0 and $X_{N+1}=f_{1}\left(X_{N}\right)$ if $X_{N-1}$ belongs to the interval 1 .

The next step was the study of the effect of perturbations. The way chosen to introduce a perturbation in the system consisted of adding or subtracting a pulse to the pump modulating signal. The reason for this choice is that it can be experimentally implemented in an easy way, even within an electronic feedback system [33]. Several numerical responses were generated and a pump pulse of fixed power was added or subtracted once every 20 modulation periods. The leading edge of the pump pulse was applied at the phase modulation 0 (called from now on "correction phase A") and the pulse duration was $5 \%$ of the modulation period. For each pulse applied, three values of $X$ are saved: the one corresponding to two periods before the pulse, $X_{-1}$; the one corresponding to the preceding period, $X_{0}$; and the value in the same period of pulse application, $X_{1}$. This way, a "shifted first-return map" was obtained by representing the whole list of $X_{1}$ values against their coupled $X_{0}$ values, while $X_{-1}$ values are useful to keep track of the layer in the first-return map with which the system would have responded if it had not been perturbed. In Fig. 7, different "shifted first-return maps" can be appreciated for several pump pulse powers.
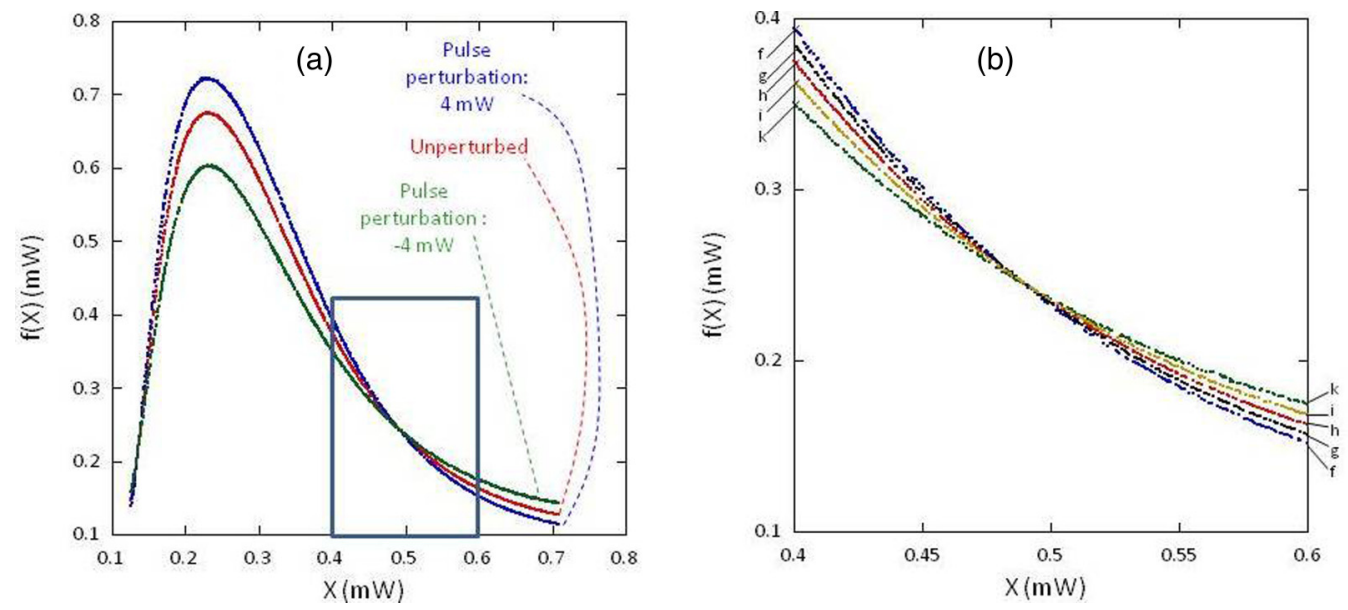

FIG. 7. (Color online) Effect of the perturbations applied in the correction phase A on the whole branch 1 of the first-return map (a) and detail around $X=0.5 \mathrm{~mW}$ (b). In (b), curves labeled with $\mathrm{f}, \mathrm{g}, \mathrm{h}, \mathrm{i}$, and k correspond to perturbation pulse powers of 4,2 , and $0 \mathrm{~mW}$ (unperturbed), and -2 and $-4 \mathrm{~mW}$, respectively. 


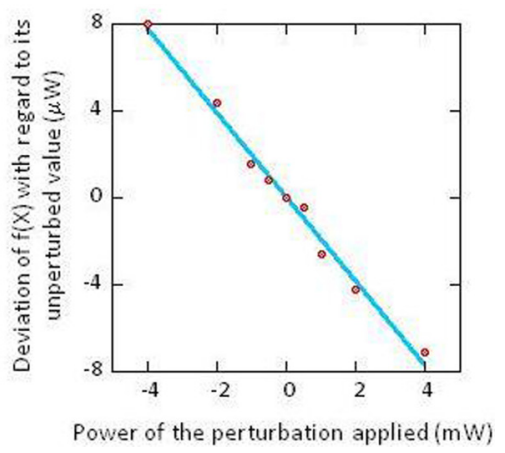

FIG. 8. (Color online) Difference between the $f(X)$ value according to the first-return map and the $f(X)$ value obtained after a perturbation applied in the correction phase $\mathrm{A}$, as a function of the modulation pulse power added to the sine-wave modulation, for different $X$ values within the bin centered at $X=0.55 \mathrm{~mW}$, and fit to a straight line.

It must be cleared up that pulses were applied once every 20 modulation periods in order that the system relaxes completely before any new perturbation. As logical, the greater the pulse power, the longer the relaxation time required. It was checked that two or three modulation periods were enough for the greatest pulse powers tested, but 20 modulation periods seemed an affordable margin which guaranteed relaxation totally.

The $X$ range was discretized into bins of $5 \times 10^{-4} \mathrm{~mW}$ of width, which were also numbered from the lowest to the greatest. The central $X$ of the $N$ th bin will be called $X_{\text {BIN_N }}$. For each bin, data for the different shifted first-return maps calculated were gathered and separated into two groups corresponding to the up and down layers, information that is contained in $X_{-1}$. For each group, displacement with regard to the original first-return map was represented against pump pulse power $(P)$ and the resulting clouds of points were fitted to straight lines, $\Delta X_{1}=\alpha_{N}^{L} P$, where $\Delta X_{1}=X_{1}-f_{A}\left(X_{\mathrm{BIN}_{-} N}\right)$ and $\alpha_{N}^{L}$ is the slope of the straight line, where $L=0,1$ depending on whether the group corresponds to the upper or the lower layer (certainly, each bin has two characteristic slopes, one per layer).

Figure 8 shows the representation corresponding to the bin to which $X=0.55 \mathrm{~mW}$ belongs and to layer 1 . It can be appreciated that the linear approximation is reasonably good for the range of pulse power considered.

This calculation also revealed that addition or subtraction of pulses with a power around $10 \mathrm{~mW}$ (or greater) turns out to be troublesome for our purposes, as the system needs more than one period to relax to its attractor. The above-mentioned straight lines are useful to calculate the effect of pulses only if the system state is close enough to its attractor. Therefore, after a pulse of circa $10 \mathrm{~mW}$ we can expect that the control procedure fails. A value of $8 \mathrm{~mW}$ is established as a limit for pulse powers to be considered small.

The next step for preparation of the control procedure consisted of dividing the $X$ range into intervals corresponding to $M$-tuples of bits, where the number of bits, $M$, had to be chosen. Its choice is crucial for success: the less the $M$ value, the simpler the method and, therefore, the faster to implement it, but on the other hand the $X$ interval corresponding to each $M$-tuple becomes greater. Note that the idea of the control method consists of continuously perturbing the system to shift its response from the $M$-tuple that would emit spontaneously to an adjacent $M$-tuple. Therefore, the greater the intervals corresponding to each $M$-tuple, the greater the power of the perturbation pulses required. As said before, in order that the system remains under control, the maximum pulse power level was established in $8 \mathrm{~mW}$. After several tests with tuples of different numbers of elements, the conclusion is that the most convenient $M$ is 8 , so that the method has been implemented for 8-tuples or "bytes": With $M$ less than 8, pulses required to drive the system turned out to be greater than the "desirable safe limit level."

Division of the $X$ range into the different byte intervals is relatively simple. A sequence $O\left(X_{0}\right)=X_{0} X_{1} \mathrm{X}_{2} \cdots X_{N \_\mathrm{MAX}}$ was generated $\left(N \_\right.$MAX $\left.=100000\right)$, which led to its corresponding sequence of bits $S\left(X_{0}\right)=K_{0} K_{1} K_{2} \cdots K_{N \_ \text {MAX }}$. For each $X_{N}$, the couple $\left\{K_{N} K_{N+1} \cdots K_{N+7}, X_{N}\right\}$ was saved (save for the last eight terms of the whole sequence, obviously). Then, the list obtained was sorted according to the byte ordering provided by the kneading theory (see Sec. II) and, within each byte, from the lowest to the highest $X$. We define $X_{\text {TARGET_N }}$ as the mean between the lowest and highest $X$ of the $N$ th byte of the list. So, the way to force the system to emit the $N$ th byte of the list was driving it to emit an $X$ as close to $X_{\text {TARGET_N }}$ as possible.

As foreseen, not all possible $2^{8}$ bytes are found, because the map presents forbidden sequences. Bytes corresponding to the smallest and greatest $X$ values found are $001^{6}$ and $1001^{5}$. According to the ordering explained in Sec. II, any byte without those limits is forbidden. It can be easily checked that this amounts to saying that any byte containing $0^{3}, 00110$, or $001^{4} 0$ is forbidden.

Finally, to facilitate calculations during application of the control method it is convenient to calculate previously the matrices $P^{L}$ (still, $L$ stands for the layer label, 0 or 1 ), whose element $P_{i j}^{L}$ is defined as

$$
P_{i j}^{L}=\frac{\left[X_{\mathrm{TARGET}_{-} j}-f_{L}\left(X_{\mathrm{BIN}_{-} i}\right)\right]}{\alpha_{i}^{L}} .
$$

These elements are the pulse powers to be applied if, after registering an emission which belongs to bin $i$, the system must be driven towards byte $j$.

\section{B. Implementation of the control method}

Once this previous study is completed, the method proposed in Refs. [6,7] can be implemented. The goal of the method consists of forcing the laser to follow a sequence of bits in "real time," certainly with an unavoidable delay: Specifically, the control system presented here has been planned to receive one random bit per laser modulation period and emit this bit eight periods afterwards.

First of all, the laser response is stabilized in the periodic orbit 1 [33]. Then, for each modulation period $i$, a random generator provides a bit value $K_{i}$ and the small feedback control pulse is applied in order to get this bit eight periods later. So, the control process starts driving the laser towards byte $1^{7} K_{1}$, then to $1^{6} K_{1} K_{2}$, etc. The way to drive the laser 


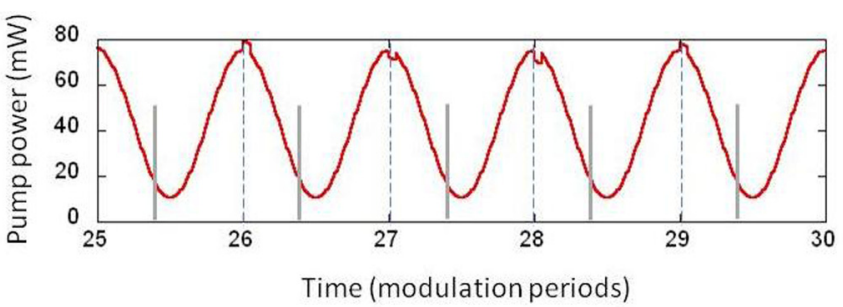

FIG. 9. (Color online) Pump modulation profile with the measurement phases pointed out by vertical solid lines and the correction phases A pointed out by vertical dashed lines. At these latter phases, pulses added or subtracted to the sine-wave modulation can also be observed.

towards the next byte is the following: Suppose that in period $N$ a value $X_{N}$ is registered at the measurement phase. The bin to which $X_{N}$ belongs is identified (bin $i$ ). The random generator provides a new bit, $K_{N+1}$, so that the laser must be driven towards the center of the byte $K_{N-6} \cdots K_{N} K_{N+1}$, whose label in the list of bytes is $j$. So, at the correction phase, a pulse whose power is $P_{i j}^{L}$ is added to pump modulation.

As said in the previous subsection, any byte containing $0^{3}, 00110$, or $001^{4} 0$ is a forbidden byte. The usual procedure to overcome occurrence of forbidden sequences was also employed here: If the random bit generator provides a sequence of bits $K_{1} \cdots K_{N-1}$, so that $K_{1} \cdots K_{N}$ is a forbidden sequence, the $N$ th bit is forced to take the value $\left(1-K_{N}\right)$. Whenever the sequence $K_{1} \cdots K_{N-1}$ is registered in the receiver stage, the next bit is identified as meaningless and is taken out of the message (expressed in terms of information theory: The value of this bit is forced by the grammar of the system and, therefore, it does not carry information).

Figure 9 illustrates the sequences of measurement and correction phases. Also, it shows the pump power profile with the perturbation pulses included, which gives an idea of the relative amount of pulses with regard to the unperturbed pump power.

However, if the method is implemented as described above, control of the bit sequences is really low. Specifically, control of the system is very troublesome whenever $X$ falls within the region around $X=0.49 \mathrm{~mW}$. Looking at Fig. 7(b), the reason why is clear: $f(X=0.49 \mathrm{~mW})$ remains almost the same, no matter the pulse perturbation power. Therefore, the $\alpha$ values of the bins around this region are very small and, according to (expression anterior), the correction pulse to be applied must be very powerful, which destabilizes the system.

In order to overcome this problem, the analysis of reaction of the system to perturbations is repeated at other correction phases. Figure 10 shows the results corresponding to $\phi=$ $0.75 \pi$ (from now on, called "correction phase B"). It is clear that in the region around $X=0.49 \mathrm{~mW}$, the perturbations do have an appreciable effect. So, the initial procedure is slightly modified: Depending on the $X$ range, the correction is calculated and applied according to data for correction phase A or B.

Figure 11 shows the dependence of the slopes $\alpha$ on $X$, for both correction phases and for layer 1 (the figure corresponding to layer 0 is not shown because it is almost exactly the same as Fig. 11). Certainly, for each $X$, the correction phase employed

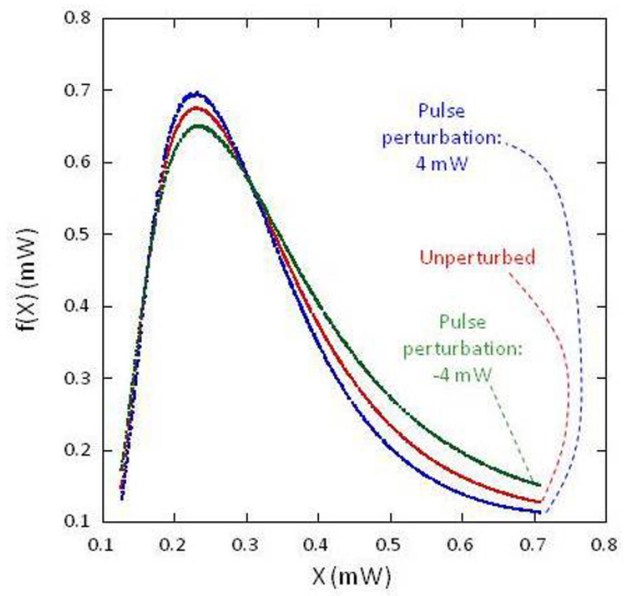

FIG. 10. (Color online) Effect of the perturbations applied in the correction phase B on the whole branch 1 of the first-return map.

is the one with greatest slope in absolute value (concretely, correction phase A is chosen if $0.145 \mathrm{~mW}<X<0.372 \mathrm{~mW}$ and correction phase B is chosen elsewhere). Table I shows an extract of a typical controlled sequence. The differences between the target $X$ values [ $X_{\text {TARGET- } j}$ in Eq. (10)] and the $X$ values emitted can be appreciated, as well as the pulse powers required for the control. The statistics of the relative differences between target and emitted $X$ values and of the pulse powers is shown in Tables II and III. This statistics has been calculated for a sequence of $10^{5}$ bits. In view of the results of Table II, the system can be considered under control. On the other hand, pulse power distribution in Table III shows how the pulse powers required do not exceed the "safe limit level" $(8 \mathrm{~mW})$, although the greatest values are near the limit. This result justifies the choice of 8-tuples (or "bytes") for control of this system: tuples of less than eight elements would require greater pulse powers, which would entail that the system would not relax on time to its attractor and the control procedure would not work; and tuples of more than eight elements would make the control process feasible with smaller pulse powers, but they would also increase complexity: mainly, the number of elements of the matrices $P^{L}$ becomes increased, which entails a slower calculation of the correction pulse power.

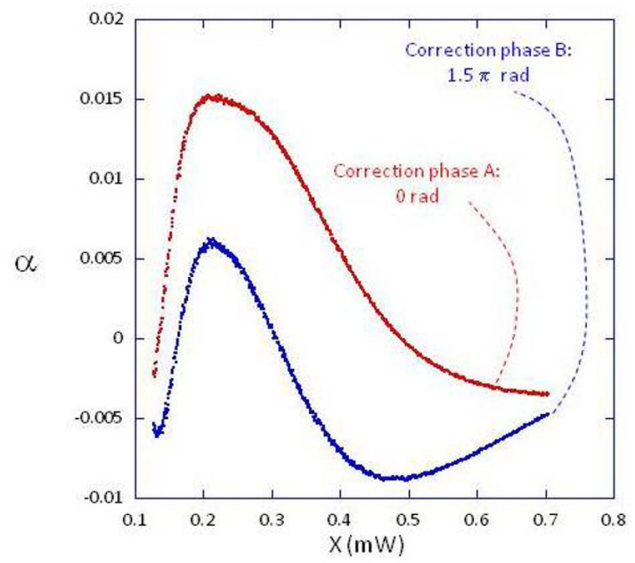

FIG. 11. (Color online) Slopes $\alpha$ as a function of $X$, for both correction phases and for layer 1 . 
TABLE I. Extract of a typical controlled sequence.

\begin{tabular}{llc}
\hline \hline $\begin{array}{l}\text { Target } X \\
(\mathrm{~mW})\end{array}$ & $\begin{array}{c}X \text { value } \\
\text { emitted }(\mathrm{mW})\end{array}$ & $\begin{array}{c}\text { Pulse power } \\
\text { applied }(\mathrm{mW})\end{array}$ \\
\hline 0.5869 & 0.5868 & 1.413 \\
0.1687 & 0.1667 & 0.3189 \\
0.5087 & 0.5160 & 2.850 \\
0.2410 & 0.2390 & -2.317 \\
0.6861 & 0.6800 & 1.144 \\
0.1313 & 0.1294 & 0.6752 \\
0.1848 & 0.1909 & -2.311 \\
0.6487 & 0.6390 & -0.9826 \\
0.1431 & 0.1419 & 0.7054 \\
0.2848 & 0.2911 & -2.0942 \\
0.6300 & 0.6324 & 0.9431 \\
0.1492 & 0.1473 & 0.0790 \\
0.3368 & 0.3348 & -3.027 \\
0.5040 & 0.5063 & -1.174 \\
0.2200 & 0.2206 & 1.055 \\
0.6861 & 0.6944 & 1.257 \\
0.1313 & 0.1291 & 0.0066 \\
0.1848 & 0.1871 & -2.903 \\
0.6487 & 0.6350 & -0.1275 \\
\hline \hline
\end{tabular}

Despite the small differences between target $X$ 's and emitted $X$ 's, there is still a problem to be solved in order to use the system as a reliable emitter of predefined bit sequences.

\section{Border trouble}

When comparing the bits provided by the random generator and the bits reproduced by the laser, some discrepancies were registered. Their occurrences coincided with some appearances of the sequence 0011. The reason why this sequence is troublesome is that its appearance necessarily requires emission of any of the bytes closest to the $0-1$ border. In effect, the eight allowed bytes closest to the border are 01001110, 01001111, 01001101, 01001100, 11001100, 11001101,11001111 , and 11001110. When the system is required to emit any of these bytes, the $X$ coordinate must be driven to the neighborhood of the border between regions 0 and 1. It is plain that, in the surroundings of the border, small deviations between targeted and emitted $X$ may mean a discrepancy between the bit required and the bit emitted. In order to secure complete correspondence between bits required and bits emitted, the solution is to forbid appearance of the

TABLE II. Histogram of the percentage differences between targeted and emitted $X$ values in a typical sequence of $10^{5}$ terms.

\begin{tabular}{lc}
\hline \hline $\begin{array}{l}\text { Absolute percentage } \\
\text { difference }\end{array}$ & $\begin{array}{c}\text { Number of occurrences } \\
\text { within the range }\end{array}$ \\
$<1 \%$ & 45530 \\
$1 \%-2 \%$ & 37862 \\
$2 \%-3 \%$ & 9819 \\
$3 \%-4 \%$ & 5322 \\
$4 \%-5 \%$ & 1328 \\
$5 \%-6 \%$ & 134 \\
$6 \%-7 \%$ & 5 \\
$>7 \%$ & 0 \\
\hline \hline
\end{tabular}

TABLE III. Histogram of the pulse powers (absolute value) required to control a typical sequence of $10^{5}$ terms.

\begin{tabular}{lc}
\hline \hline $\begin{array}{l}\text { Pulse power } \\
\text { range }(\mathrm{mW})\end{array}$ & $\begin{array}{c}\text { Number of occurrences } \\
\text { within the range }\end{array}$ \\
\hline$<1$ & 58115 \\
$1-2$ & 25140 \\
$2-3$ & 14107 \\
$3-4$ & 2116 \\
$4-5$ & 402 \\
$5-6$ & 103 \\
$6-7$ & 17 \\
$>7$ & 0 \\
\hline \hline
\end{tabular}

sequence 0011 by adding an "artificial" rule to the "natural" grammar of the system: after the sequence 00 , the meaningless sequence 10 will be added. In fact, this rule excludes all forbidden bytes (note: bytes containing $0^{3}, 00110$, or $001^{4} 0$ ), so that the whole grammar considered becomes reduced to this simple rule.

Once this rule is incorporated, correspondence between bits requested and bits emitted is perfect in all the tests performed, which consisted of five series with $10^{7}$ bits.

\section{INFLUENCE OF NOISE IN THE CONTROL}

In a real situation, signal must be measured with a detector affected by noise. In order to analyze completely the possibilities of the system, it has been considered convenient to simulate its performance also considering the influence of noise in the process. Therefore, different numerical simulations have been performed under different signal to noise ratio (SNR) assumptions, where this parameter is defined, as usual, as

$$
\begin{aligned}
\mathrm{SNR}= & \text { Average laser output power } / \\
& \text { Signal standard deviation. }
\end{aligned}
$$

In order to consider the effect of noise, the signal detected has been considered the sum of the signal calculated by numerical simulation plus a random quantity, whose probability

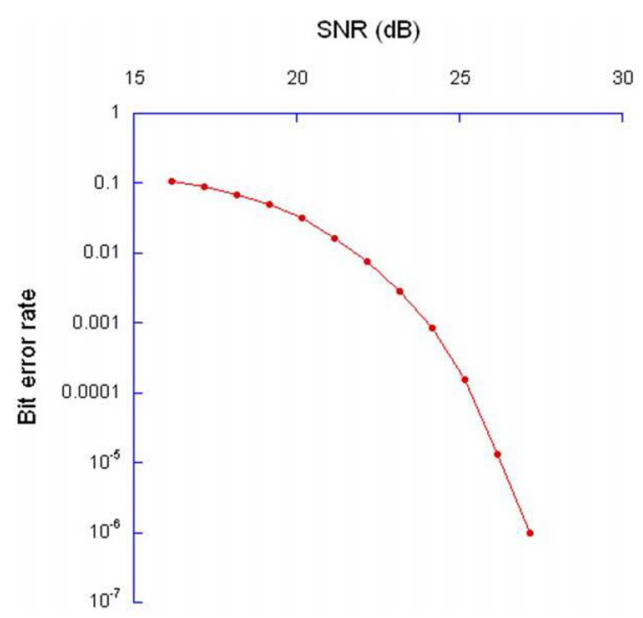

FIG. 12. (Color online) BER as a function of SNR for the digital encoding procedure under analysis. 
distribution is a Gaussian whose half width is the standard deviation. For each SNR considered, a random sequence of $10^{6}$ bits has been generated and the number of wrong bits emitted by the laser has been counted in order to calculate the bit error rate (BER) (if the number of wrong bits was less than 10 , the process was repeated with $10^{7}$ bits). Figure 12 shows the results obtained. For SNR greater than $27.2 \mathrm{~dB}$, the sequences of $10^{7}$ bits were reproduced by the laser exactly. The trend that can be appreciated in Fig. 12, which is quite a typical trend for BER against standard deviation [34], seems to point out that for $\mathrm{SNR}=30 \mathrm{~dB}$ the BER is in the $10^{-10}-10^{-9}$ range or even lower than that. Therefore, counting on an SNR not especially demanding, a BER can be obtained whose value is low enough for a good deal of current communication standards.

\section{CONCLUSIONS}

Control by the Hayes technique can be applied to an EDFL successfully by perturbing its sine-wave modulation power with pulses whose power ranges are easily manageable and whose required precision is perfectly controllable with currently available devices. The general method by Hayes must be customized for a successful control of the system. Mainly, the effect on the system behavior of a change in the control parameter can be really small for a certain range of the system's dynamic variable, which makes the system control very troublesome whenever the variable value falls within that range. In the present work it has been shown that application of the perturbation at two different modulation phases depending on the $X$ range suffices to guarantee the control of the system.

Even though the system can be considered under control, differences between bits required and bits emitted can be obtained whenever the dynamic variable must be driven near the border between regions of the symbolic partition. In order to avoid this, it is convenient to introduce additional grammar rules which forbid some sequences whose appearance force the system to get too close to the border.

The study of the system performance as a function of noise shows that a detection system with quite a conventional SNR would suffice to obtain low enough BER values to meet many of the current communication standards.

\section{ACKNOWLEDGMENTS}

The author expresses thanks for the suggestions and remarks of the referees that have contributed to improve the paper. Thanks as well to Dr. Roberto Barrio for helpful discussions. This work was supported by the Spanish Ministry of Economy and Competitiveness (FIS2010-20821 project) and by the Diputación General de Aragón.
[1] L. M. Pecora and T. L. Carroll, Phys. Rev. Lett. 64, 821 (1990).

[2] K. M. Cuomo and A. V. Oppenheim, Phys. Rev. Lett. 71, 65 (1993).

[3] P. Colet and R. Roy, Opt. Lett. 19, 2056 (1994).

[4] C. R. Mirasso, P. Colet, and P. García-Fernández, IEEE Photonics Technol. Lett. 8, 299 (1996).

[5] G. D. Van Wiggeren and R. Roy, Phys. Rev. Lett. 81, 3547 (1998).

[6] S. Hayes, C. Grebogi, E. Ott, and A. Mark, Phys. Rev. Lett. 73, 1781 (1994).

[7] S. Hayes, C. Grebogi, and E. Ott, Phys. Rev. Lett. 70, 3031 (1993).

[8] E. Ott, C. Grebogi, and J. A. Yorke, Phys. Rev. Lett. 64, 1196 (1990).

[9] E. M. Bollt and M. Dolnik, Phys. Rev. E 55, 6404 (1997); M. Dolnik and E. M. Bollt, Chaos 8, 702 (1998).

[10] V. Dronov, M. R. Hendrey, T. M. Antonsen, and E. Ott, Chaos 14, 30 (2004).

[11] J. Used and J. C. Martín, Phys. Rev. E 87, 052921 (2013).

[12] E. M. Bollt and Y. C. Lai, Phys. Rev. E 58, 1724 (1998).

[13] E. M. Bollt, Int. J. Bifurcation Chaos 13, 269 (2003).

[14] R. Gilmore and M. Lefranc, The Topology of Chaos (Wiley, New York, 2011).

[15] J. Used and J. C. Martín, Phys. Rev. E 79, 046213 (2009).

[16] B. L. Hao, Elementary Symbolic Dynamics and Chaos in Dissipative Systems (World Scientific, Singapore, 1989).

[17] B. L. Hao, Phys. D 51, 161 (1991).

[18] J. Milnor and R. Thurston, in Dynamical Systems, Lecture Notes in Math Vol. 1342 (Springer, Berlin, 1988), p. 465-563.

[19] R. L. Devaney, An Introduction to Chaotic Dynamical Systems (Westview Press, Boulder, CO, 2003).
[20] C. E. Shannon and W. Weaver, The Mathematical Theory of Communication (University of Illinois Press, Champaign, IL, 1964).

[21] B. Kitchens, Symbolic Dynamics. One-sided, two-sided and Countable State Markov Shifts (Springer-Verlag, Berlin, 1998).

[22] D. Lind and B. Marcus, An Introduction to Symbolic Dynamics and Coding (Cambridge University Press, Cambridge, 1996).

[23] P. Cvitanovic, G. H. Gunaratne, and I. Procaccia, Phys. Rev. A 38, 1503 (1988).

[24] I. J. Sola, J. C. Martín, and J. M. Álvarez, Opt. Commun. 212, 359 (2002).

[25] J. Used and J. C. Martín, Phys. Rev. E 82, 016218 (2010).

[26] E. M. Bollt, T. Stanford, Y. C. Lai, and K. Zyczkowski, Phys. Rev. Lett. 85, 3524 (2000); Phys. D 154, 259 (2001).

[27] J. Gluckenheimer and P. J. Holmes, Nonlinear Oscillations, Dynamical Systems and Bifurcations of Vector Fields (Springer, Berlin, 1983).

[28] A. Katok and B. Hasselblatt, Introduction to the Modern Theory of Dynamical Systems (Cambridge University Press, Cambridge, 1995).

[29] R. Badii and A. Politi, Complexity: Hierarchical Structures and Scaling in Physics, Cambridge Nonlinear Science Series Vol. 6 (Cambridge University Press, Cambridge, 1997).

[30] Y. C. Lai, E. Bollt, and C. Grebogi, Phys. Lett. A 255, 75 (1999).

[31] J. Plumecoq and M. Lefranc, Phys. D 144, 231 (2000).

[32] J. Kurths, A. Voss., P. Saparin, A. Witt, H. J. Kleiner, and N. Wessel, Chaos 5, 88 (1995).

[33] S. Bielawski, D. Derozier, and P. Glorieux, Phys. Rev. A 47, R2492 (1993).

[34] J. Proakis and M. Salehi, Digital Communications (McGrawHill Education, New York, 2007). 